\title{
New Solitary-wave Solutions for the Higher Order Nonlinear Schrödinger Equation with Both Real and Imaginary Raman Terms
}

\author{
Woo-Pyo Hong \\ Department of Physics, Catholic University of Daegu, Hayang, Kyongsan, \\ Kyungbuk 712-702, South Korea
}

Reprint requests to Prof. Dr. W.-P. H.; E-mail:wphong@mail.cu.ac.kr.

Z. Naturforsch. 58a, $667-671$ (2003); received October 2, 2003

\begin{abstract}
We find new solitary-wave solutions of the higher order nonlinear Schrödinger equation with both real and imaginary Raman terms, which can model an ultrashort pulse propagation through optical fibers, under some constraint among the model coefficients. The physical conditions such as the wavelength needed to launch the pulse, the types of optical fibers, and the required peak power, are obtained from the constraints for the solitary-wave solutions. - PACS number(s): 42.65.Tg, 42.81Dp, 02.30.Jr, 42.79.Sz.
\end{abstract}

Key words: Extended Higher-order Nonlinear Schrödinger Equation; Analytic Bright Solitary-wave Solution; Solitary-wave Propagation.

\section{Introduction}

Since optical solitons have been predicted by Hasegawa and Tappert [1] and experimentally observed by Mollenauer et al. [2], it has been regarded as the next generation technology for high-capacity optical communications, chiefly because of its promise to transmit signals over long distances while resisting chromatic dispersion $[3,4]$. The dynamics of a short optical soliton can be well described in the context of the Nonlinear Schrödinger equation (NLSE), which has only the self-phase modulation (SPM) and group velocity dispersion (GVD) terms $[1,3,4]$. However, if one studies the properties of much shorter optical solitons in the subpicosecond to femtosecond domain, it is necessary to include the higher order effects such as the third order dispersion (TOD), the nonlinear dispersion of the Kerr coefficient (Kerr dispersion), and the stimulated Raman scattering (SRS) which induces the self-frequency shift of the NLSE soliton [5]. As such a model, many authors (see $[4,6]$ for details) have adopted the higher order nonlinear Schrödinger equation (HONLSE) in the form

$$
\begin{aligned}
& i E_{\xi}+\alpha_{1} E_{\tau \tau}+\alpha_{2}|E|^{2} E= \\
& \quad i \alpha_{3} E_{\tau \tau \tau}+i \alpha_{4}\left(|E|^{2} E\right)_{\tau}+i \alpha_{5} E\left(|E|^{2}\right)_{\tau},
\end{aligned}
$$

where $E(\xi, \tau)$ is the slowly varying envelope of the electric filed, the subscripts $\xi$ and $\tau$ are the nor- malized spatial and temporal partial derivatives, and $\alpha_{1}, \alpha_{2}, \alpha_{3}, \alpha_{4}$, and $\alpha_{5}$ are the coefficients related to GVD, SPM, TOD, self-steepening, and SRS, respectively. Some types of exact analytic dark, bright, kink solitary-waves, and bright $\mathrm{N}$-soliton solutions for the special values of the coefficients have been found $[4,6]$.

However, in the previous works (see [4] for a detailed summary), the possibility of the SRS coefficient being complex has not been considered: $\alpha_{5}=\nu_{5}+i \mu_{5}$ can be a complex number with both real and imaginary Raman terms. The first term, $\nu_{5}$, is due to the frequency dependent gain coefficient, which is generally small enough to be ignored. The $\mu_{5}$ coefficient comes from the nonlinear dissipation due to the Raman process in the fiber [3,4], so it can not be ignored for a femtosecond pulse propagation. Thus, (1) should be modified as

$$
\begin{aligned}
& i E_{\xi}+\alpha_{1} E_{\tau \tau}+\alpha_{2}|E|^{2} E= \\
& i \alpha_{3} E_{\tau \tau \tau}+i \alpha_{4}\left(|E|^{2} E\right)_{\tau}+i\left(\nu_{5}+\mu_{5}\right) E\left(|E|^{2}\right)_{\tau} .
\end{aligned}
$$

For the case $\nu_{5}=0$ we encounter a physically more appropriate HONLSE than previously considered by Agrawal and Headley [7]. Meanwhile, (2) without the real Raman term, i.e. $\mu_{5}=0$, or (1), is mathematically interesting since self-consistent $\mathrm{N}$-soliton solutions have been found by many authors [4]. However, if the real Raman term is included, then it leads to a spectral redshift acting as an effective loss term so that 
$\mathrm{N}$-soliton or solitary-wave solutions can not exist [8]. Mathematically speaking, the HONLSE with the real Raman term can not be reduced to an integrable ordinary differential equation by using the point-symmetry group transformations as know as "symmetry reduction method" without further imposing a restriction on $\mu_{5}$, i.e., $\mu_{5}=0$, which is only achieved for the instantaneous Raman response [9]. The existence of the solitary-wave solution of the HONLSE with both real and imaginary Raman terms (the extended HONLSE) have not been investigated, which will be pursued in the present work.

The paper is organized as follows. In Sect. 2, by applying a simple ansatz method, we show that there exist analytic bright solitary-wave solutions of the extended HONLSE under some physical constraints on the model coefficients, which lead to the range of wavelengths required for the solutions, the types of optical fibers, and the peak power. The conclusions are followed in Section 3 .

\section{Analytic Bright Solitary-wave Solutions}

Based on the notations of Hasegawa [3] and Agrawal [4], we rewrite the extended HONLSE in (2) with the dimensionless real coefficients in terms of the fiber optics parameters [7]

$i E_{\xi}+\alpha_{1} E_{\tau \tau}+\alpha_{2}|E|^{2} E=$

$i \alpha_{3} E_{\tau \tau \tau}-i \alpha_{4}\left(|E|^{2} E\right)_{\tau}+\left(\alpha_{5}+i \alpha_{6}\right) E\left(|E|^{2}\right)_{\tau}$,

where $\alpha_{1}=-\frac{1}{2} \beta_{2} /\left|\beta_{2}\right|, \alpha_{2}=N^{2}=\gamma P_{0} T^{2} /\left|\beta_{2}\right|$, $\alpha_{3}=\beta_{3} /\left(6\left|\beta_{2}\right| T\right), \alpha_{4}=2 N^{2} /\left(\omega_{0} T\right), \alpha_{5}=$ $N^{2}\left(T_{\mathrm{R}} / T\right), \alpha_{6}=\epsilon \alpha_{5}$ with $\epsilon \lesssim 1$ since it originates from the SRS term, $\left.\tau=\widetilde{(t}-z / v_{g}\right) / T, \xi=$ $\left(z\left|\beta_{2}\right|\right) / T^{2}$, and $E(\xi, \tau)=A(\xi, \tau) / P_{0}^{1 / 2}$, where $A(\xi, \tau)$ is a slowly varying amplitude. The parameters $v_{g}, \beta_{2}, \beta_{3}, T$, and $P_{0}$ denote, group velocity, GVD, TOD, the pulse width defined as $T_{\mathrm{FWHM}} \equiv 1.763 T$, and the peak power of the pulse, respectively. The coefficient $T_{\mathrm{R}}$ is related to the slope of the Raman-gain spectrum near the carrier frequency $\omega_{0}$, and $\gamma$ is the nonlinearity coefficient (see [4,7] for detail). In fact, for the case of $\alpha_{6}=0$, the analytic kink-type solitarywave solutions for (3) were obtained by Agrawal and Headley [7] and their dynamical behaviors are numerically investigated. However, no self-consistent bright solitary-wave solution has been shown to exist [8].

Here we are interested in finding traveling solitarywave solutions of (3) by setting an ansatz function.
The order of which is calculated as $N=1$ from the balance act between the highest nonlinear dispersion terms $\left(\alpha_{4}, \alpha_{5}\right.$, and $\left.\alpha_{6}\right)$ and the TOD term $\left(\alpha_{3}\right)$, i.e., $3 N+1=N+3$, in the form

$$
E(\xi, \tau)=E_{0} \operatorname{sech}(\tau+\chi \xi) e^{i(K \xi-\Omega \tau)},
$$

where $E_{0}$ is taken to be a complex amplitude. The dimensionless real parameters $\Omega, \chi$ and $K$ represent the shift from the carrier frequency $\omega_{0}$, the shift from the group velocity, and the corresponding change in the propagation constant, respectively [6]. By substituting (4) into (3), expanding tanh terms to sech terms, and equating the coefficients terms containing independent combinations of tanh and sech functions to zero, we obtain the following 4 independent complex parametric equations

$$
\begin{aligned}
& \alpha_{3} \Omega^{3}-\alpha_{1} \Omega^{2}+\left(E_{0}^{2} \alpha_{4}+3 \alpha_{3}\right) \Omega \\
& \quad+E_{0}^{2} \alpha_{2}-K-\alpha_{1}=0, \\
& \left(3 \alpha_{4}-2 \alpha_{6}+2 i \alpha_{5}\right) E_{0}^{2}+3 \alpha_{3} \Omega^{2}-2 \alpha_{1} \Omega \\
& \quad+\chi+5 \alpha_{3}=0, \\
& \quad\left(E_{0}^{2} \alpha_{4}+6 \alpha_{3}\right) \Omega+E_{0}^{2} \alpha_{2}-2 \alpha_{1}=0, \\
& \left(3 \alpha_{4}-2 \alpha_{6}+2 i \alpha_{5}\right) E_{0}^{2}+6 \alpha_{3}=0 .
\end{aligned}
$$

From (7) and (8), we find $E_{0}$ and $\Omega$ as

$$
\begin{aligned}
& E_{0}= \pm \sqrt{\frac{-6 \alpha_{3}}{\left(3 \alpha_{4}+2 i \alpha_{5}-2 \alpha_{6}\right)}}, \\
& \Omega=\frac{\left(3 \alpha_{2} \alpha_{3}+3 \alpha_{1} \alpha_{4}+2 i \alpha_{1} \alpha_{5}-2 \alpha_{1} \alpha_{6}\right)}{6 \alpha_{3}\left(\alpha_{4}+i \alpha_{5}\right)},
\end{aligned}
$$

and from (5) and (6), we find $K\left(E_{0}, \Omega\right)=$ $K_{\mathrm{re}}\left(E_{0}, \Omega\right)+i K_{\mathrm{im}}\left(E_{0}, \Omega\right)$ and $\chi\left(E_{0}, \Omega\right)=$ $\chi_{\mathrm{re}}\left(E_{0}, \Omega\right)+i \chi_{\mathrm{im}}\left(E_{0}, \Omega\right)$, of which lengthy expressions are omitted here. As $K, \chi$, and $\Omega$ are assumed to be real for physical solutions, we require both $K_{\mathrm{im}}=0$ and $\chi_{\mathrm{im}}=0$, which results in the two additional constraints

$$
\left(\alpha_{1} \alpha_{4}+3 \alpha_{2} \alpha_{3}\right)^{2} \cdot \mathcal{F}\left(\alpha_{i}\right)=0
$$

and

$$
\left(\alpha_{1} \alpha_{4}+3 \alpha_{2} \alpha_{3}\right)^{3} \cdot \mathcal{G}\left(\alpha_{i}\right)=0,
$$

respectively, where $\mathcal{F}\left(\alpha_{i}\right)=\left(3 \alpha_{4}-2 \alpha_{6}\right) \cdot(\ldots)$ is a complex polynomial of $\alpha_{i}$, and $\mathcal{G}\left(\alpha_{i}\right)=\left(\alpha_{4}-\right.$ 
$\left.\alpha_{6}\right)\left(\alpha_{4}-\alpha_{6}+i \alpha_{5}\right)^{2}$. Therefore we obtain the necessary constraint for the bright solitary-wave solution of (3) as

$$
\alpha_{1} \alpha_{4}+3 \alpha_{2} \alpha_{3}=0 \Rightarrow \alpha_{4}=-\frac{3 \alpha_{2} \alpha_{3}}{\alpha_{1}}>0
$$

since $\alpha_{4}$ is a non-negative real number. Furthermore, as $\alpha_{2}=N^{2}>0$, it implies that

$$
\alpha_{3} / \alpha_{1}<0 \text { or } \beta_{2} \beta_{3}>0 \text {. }
$$

After substituting $\alpha_{3}=-\alpha_{1} \alpha_{4} / 3 \alpha_{2}$ from (12) into (4) and expressing the complex amplitude in polar coordinates,

$$
E(\xi, \tau)=\left|E_{0}^{\prime}\right| \operatorname{sech}(\tau+\chi \xi) e^{i(K \xi-\Omega \tau+\phi)},
$$

we find

$$
\begin{aligned}
& \left|E_{0}^{\prime}\right|=\sqrt{\frac{2 \alpha_{1} \alpha_{4}}{\alpha_{2}\left[\left(3 \alpha_{4}-2 \alpha_{6}\right)^{2}+4 \alpha_{5}^{2}\right]^{1 / 2}}}, \\
& \chi=-\frac{\alpha_{1}\left(\alpha_{4}^{2}+3 \alpha_{2}^{2}\right)}{3 \alpha_{2} \alpha_{4}} \\
& K=\frac{2 \alpha_{1} \alpha_{2}^{2}}{3 \alpha_{4}^{2}}, \quad \Omega=\frac{\alpha_{2}}{\alpha_{4}} \\
& \phi=-\tan ^{-1}\left(\frac{2 \alpha_{5}}{3 \alpha_{4}-2 \alpha_{6}}\right) .
\end{aligned}
$$

Further restrictions on $\beta_{2}$ and $\beta_{3}$ are as follows: For having a real amplitude $\left|E_{0}^{\prime}\right|, \beta_{2}<0$ is required from $\alpha_{1}=-\frac{1}{2} \beta_{2} /\left|\beta_{2}\right|>0$, and with the $\beta_{2} \beta_{3}>0$ constraint from (13) we find

$$
\beta_{2}<0 \text { and } \beta_{3}<0 \text {. }
$$

We note that the usual graded-index fibers do not support the bright solitary-wave solution found in (14) since in such fibers $\beta_{3}$ is always positive while $\beta_{2}$ is negative [4]. However, the constraint in (16) can be realized in the dispersion-flattened optical fibers having low dispersions over a wavelength range of about $1.3-1.6 \mu \mathrm{m}$ by using multiple (double and quadruple) cladding layers [4]. In the following analysis, we adopt the fitted dispersion coefficients

$$
\begin{aligned}
\beta_{2}(\lambda)= & -392.3+1140.2 \lambda-1013.8 \lambda^{2} \\
& +284.1 \lambda^{3}(\mathrm{ps})^{2} / \mathrm{km} \\
\beta_{3}(\lambda)= & -5.3052 \times 10^{-5} \lambda^{2}
\end{aligned}
$$

$$
\cdot\left(1140.2-2027.6 \lambda+852.3 \lambda^{2}\right)(\mathrm{ps})^{3} / \mathrm{km}
$$

Table 1. The coefficients corresponding to the four different wavelengths toward the ZDW using the physical parameters $N=1, T=50$ fs, $T_{\mathrm{R}}=3 \mathrm{fs}, \gamma=3 \mathrm{~W}^{-1} \mathrm{~km}^{-1}$, and $\epsilon=0.1$ which yield $\alpha_{1}=0.5, \alpha_{2}=1.0, \alpha_{5}=0.06$, and $\alpha_{6}=0.006$.

\begin{tabular}{lcccc}
\hline$\lambda$ & $\alpha_{3}$ & $\alpha_{4}$ & $\left|E_{0}^{\prime}\right|$ & $P_{0}$ (watt) \\
\hline $0.922 \lambda_{\text {ZDW }}$ & -0.0026 & 0.0156 & 0.5163 & 595.8 \\
$0.975 \lambda_{\text {ZDW }}$ & -0.0847 & 0.0495 & 0.5219 & 325.7 \\
$0.995 \lambda_{\text {ZDW }}$ & -0.5374 & 0.0505 & 0.5239 & 76.4 \\
$0.999 \lambda_{\text {ZDW }}$ & -2.7944 & 0.0507 & 0.5243 & 15.7 \\
\hline
\end{tabular}
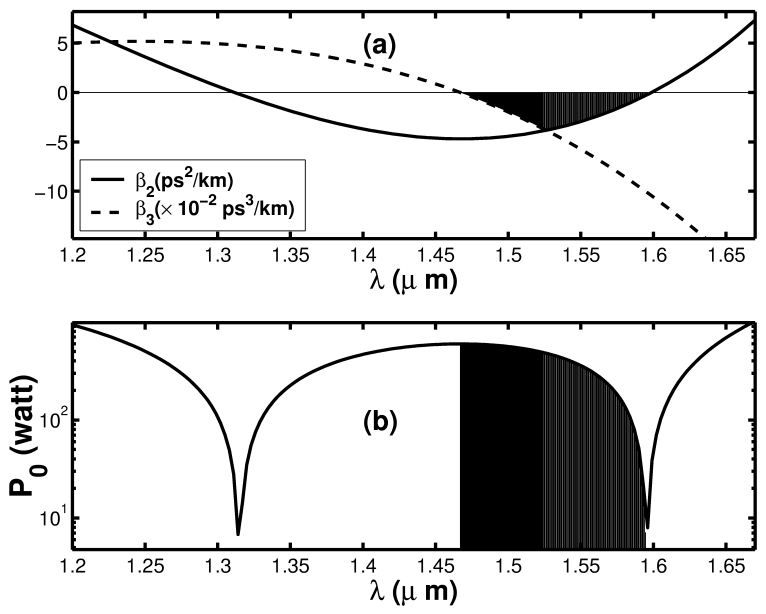

Fig. 1. (a) Plot of $\beta_{2}(\lambda)$ and $\beta_{3}(\lambda)$ in (17), which are fitted to the experimentally measured data in [10] as the dispersion coefficients of the quadruple-clad fiber. The filled area represents the wavelength for $\beta_{2}<0$ and $\beta_{3}<0$ in $1.467 \mu \mathrm{m}<\lambda<1.595 \mu \mathrm{m}$. (b) The peak power required to launch a solitary wave, $P_{0}=N^{2}\left|\beta_{2}\right| /(\gamma T)$, as a function of $\lambda$ for $N=1, \gamma=3 \mathrm{~W}^{-1} \mathrm{~km}^{-1}$, and $T=50 \mathrm{fs}$.

to the experimentally measured values of the quadruple-cladding fiber [10]. Figure 1(a) shows the wavelength range for both $\beta_{2}$ and $\beta_{3}$ being negative, i.e., $1.467 \mu \mathrm{m}<\lambda<1.595 \mu \mathrm{m}$ (filled region), and the two zero-dispersion wavelengths (ZDWs) at $\lambda_{\mathrm{ZDW}, 1}=1.315 \mu \mathrm{m}$ and $\lambda_{\mathrm{ZDW}, 2}=1.595 \mu \mathrm{m}$. Figure 1(b) plots the wavelength dependent peak power, $P_{0}(\lambda)=N^{2}\left|\beta_{2}\right| /\left(\gamma T^{2}\right)$ for $N=1, \gamma=$ $3 \mathrm{~W}^{-1} \mathrm{~km}^{-1}, T_{\mathrm{R}}=3 \mathrm{fs}$, and $T=30 \mathrm{fs}$, showing the two minima at the ZDWs at which launching a solitary-wave is the most advantageous. Dynamics of solitary-waves launched near the ZDW in the context of the modified nonlinear Schrödinger equation (MNLSE) has been previously investigated by several authors, see [11]. The meaning of (12) can be more physically interpreted by expressing it in terms of the 


\begin{tabular}{llll}
\hline & $\left|E_{0}^{\prime}\right|$ & Auxiliary constraint & Wavelength constraint \\
\hline I & $\sqrt{2 \alpha_{1} \alpha_{4} / \alpha_{2}\left(\alpha_{4}^{2}+4 \alpha_{5}^{2}\right)^{1 / 2}}$ & $\alpha_{4}=\alpha_{6} \neq 0$ & $\beta_{2}(\lambda) / \beta_{3}(\lambda)=1 /\left(\epsilon T_{\mathrm{R}}\right)$ \\
II & $\sqrt{2 \alpha_{1} / 3 \alpha_{2}}$ & $\alpha_{5}=\alpha_{6}=0$ & $\beta_{2}(\lambda) / \beta_{3}(\lambda)=\omega_{0}$ \\
III $\quad\left|E_{0}^{\prime}\right|=\sqrt{2 \alpha_{1} \alpha_{4} / \alpha_{2}\left(9 \alpha_{4}^{2}+4 \alpha_{5}^{2}\right)^{1 / 2}}$ & $\alpha_{5} \neq 0, \alpha_{6}=0$ & $\beta_{2}(\lambda) / \beta_{3}(\lambda)=\omega_{0}$ \\
IV $\quad\left|E_{0}^{\prime}\right|=\sqrt{\alpha_{1} \alpha_{4} / \alpha_{2} \alpha_{5}}$ & $3 \alpha_{4}=2 \alpha_{6} \neq 0$ & $\beta_{2}(\lambda) / \beta_{3}(\lambda)=2 /\left(3 \epsilon T_{\mathrm{R}}\right)$ \\
\hline
\end{tabular}

Table 2. Four solitary-wave solutions obtained by applying auxiliary constraints, i.e. $\mathcal{F}\left(\alpha_{i}\right)=0$ or $\mathcal{G}\left(\alpha_{i}\right)=0$. dispersion coefficients

$$
\omega_{0}=2 \frac{\beta_{2}(\lambda)}{\beta_{3}(\lambda)}
$$

from which we calculate the wavelength required for a existence of the solitary-wave solution in (14) as $\lambda_{\text {sol }} \equiv 0.922 \lambda_{\mathrm{ZDW}, 2} \simeq 1.475 \mu \mathrm{m}$ for the case of the quadruple-cladding fiber (in the following we set $\left.\lambda_{\mathrm{ZDW}} \equiv \lambda_{\mathrm{ZDW}, 2}\right)$. Thus, exact solitary-wave solutions exist only near the ZDW (at 92\% of ZDW), however, by adjusting the geometry and material of the multicladding fibers, one may be able to find the wavelength required by (18) as close as to the ZDW. Table 1 shows the coefficients corresponding to four different wavelengths toward the ZDW using the physical parameters as $N=1, T=50 \mathrm{fs}, T_{\mathrm{R}}=3 \mathrm{fs}, \gamma=3 \mathrm{~W}^{-1} \mathrm{~km}^{-1}$, and $\epsilon=0.1$ which yield $\alpha_{1}=0.5, \alpha_{2}=1.0, \alpha_{5}=$ 0.06 , and $\alpha_{6}=0.006$.

Several interesting solitary-wave solutions can be obtained by applying auxiliary constraints, i.e., $\mathcal{F}\left(\alpha_{i}\right)=0$ or $\mathcal{G}\left(\alpha_{i}\right)=0$, which give wavelength constraints different from (18).

Firstly, we consider $\mathcal{G}\left(\alpha_{i}\right)=0$ which yields four special cases: (i) $\alpha_{6}=\alpha_{4} \neq 0$; (ii) $\alpha_{6}=\alpha_{5}=$ 0; (iii) $\alpha_{5}=0$ and $\alpha_{6} \neq 0$; (iv) $\alpha_{5} \neq 0$ and $\alpha_{6}=0$. For the case of (i), the amplitude is $\left|E_{0}^{\prime}\right|=$ $\sqrt{2 \alpha_{1} \alpha_{4} / \alpha_{2}\left(\alpha_{4}^{2}+4 \alpha_{5}^{2}\right)^{1 / 2}}$ and the requirement for $\lambda_{\text {sol }}$ can be found from $\beta_{2}(\lambda) / \beta_{3}(\lambda)=1 /\left(\epsilon T_{\mathrm{R}}\right)$, indicating that the wavelength decreases away from the ZDW, and the peak power to launch that the solitarywave increases as $\epsilon$ decreases. For the case of (ii), i.e., excluding the SRS term from (3) (equivalent to the MNLSE [12]), we find a new solitary-wave solution for the MNLSE with amplitude $\left|E_{0}^{\prime}\right|=\sqrt{2 \alpha_{1} / 3 \alpha_{2}}$, which is different from the solutions of Ref. [12]. For the case of (iii), we obtain a new solitary-wave solution of (1) with $\left|E_{0}^{\prime}\right|=\sqrt{2 \alpha_{1} \alpha_{4} / \alpha_{2}\left|3 \alpha_{4}^{2}-2 \alpha_{6}^{2}\right|}$ and $\phi=0$, which is different from the previous ones as summarized in [4]. For the case of (iv), in addition to the kink-type solitary wave solution in [7], we find new bright solitary-wave solution with $\left|E_{0}^{\prime}\right|=$
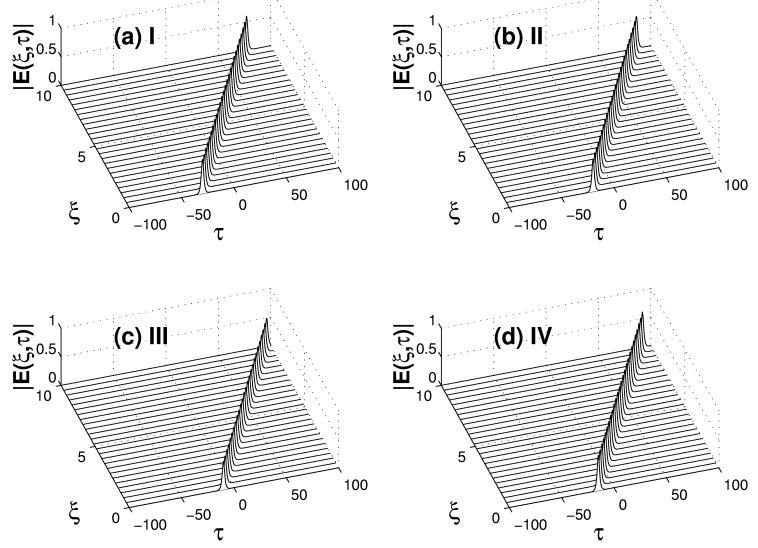

Fig. 2. Plots of the four solitary-wave solution in Table 2 for $N=1, T=50 \mathrm{fs}, T_{\mathrm{R}}=3 \mathrm{fs}, \gamma=3 \mathrm{~W}^{-1} \mathrm{~km}^{-1}, \epsilon=$ 0.1 , and $\lambda=0.9204 \lambda_{\mathrm{ZDW}}$. The amplitudes in (a) $-(\mathrm{d})$ are $0.6024,0.5774,0.5040$, and 0.6241 , respectively.

$\sqrt{2 \alpha_{1} \alpha_{4} / \alpha_{2}\left(9 \alpha_{4}^{2}+4 \alpha_{5}^{2}\right)^{1 / 2}}$ under the constraints of (16) and (18).

Secondly, by setting $\mathcal{F}\left(\alpha_{i}\right)=0$, with the auxiliary constraint $3 \alpha_{4}=2 \alpha_{6}$, i.e., $\beta_{2}(\lambda) / \beta_{3}(\lambda)=2 /\left(3 \epsilon T_{\mathrm{R}}\right)$, we obtain a new solitary-wave solution with $\left|E_{0}^{\prime}\right|=$ $\sqrt{\alpha_{1} \alpha_{4} / \alpha_{2} \alpha_{5}}$ and $\phi=-\frac{\pi}{2}$, where the wavelength constraint can vary according to $\epsilon$ and the Raman slope $T_{\mathrm{R}}$. Table 2 shows the summary of the solitary-wave solutions.

Finally, as an example, we plot in Fig. 2 the analytic solitary-wave solutions listed in Table 1 for $N=1$, $T=50$ fs, $T_{\mathrm{R}}=3 \mathrm{fs}, \gamma=3 \mathrm{~W}^{-1} \mathrm{~km}^{-1}, \epsilon=0.1$, and $\lambda=0.9204 \lambda_{\mathrm{zDW}}$. The corresponding peak amplitudes for (I) - (IV) are 0.6024, 0.5774, 0.5040, and 0.6241 , respectively. Detailed numerical stability analysis is needed to confirm the application of the solitarywave solutions to the long-haul optical system, which will be pursued in the future.

\section{Conclusions}

In conclusion, we have found new analytic bright solitary-wave solutions in (14) and (15) for the 
HONLSE with both the real and imaginary Raman terms under the necessary constraint among the model coefficients in (12) and (16), from which physical parameters such as the wavelength needed, the optical fiber type, and the required peak power, can be obtained. Depending on the auxiliary conditions, $\mathcal{F}\left(\alpha_{i}\right)=0$ or $\mathcal{G}\left(\alpha_{i}\right)=0$, we found several interesting bright solitary-wave solutions as summarized in Table 2. In particular, we have shown that the analytic bright solitary-wave solution of (3) in the limit of

[1] A. Hasegawa and F. Tappert, Appl. Phys. Lett. 23, 142 (1973); 23, 1171 (1973).

[2] L. F. Mollenauer, R. H. Stolen, and J. P. Gordon, Phys. Rev. Lett. 45, 1095 (1980).

[3] A. Hasegawa and K. Kodama, Solitons in Optical Communications, Oxford University Press, New York 1995.

[4] G. P. Agrawal, Nonlinear Fiber Optics, third ed., Academic Press, New York, 2001.

[5] F. M. Mitschke and L. F. Mollenauer, Opt. Lett. 11, 659 (1986).

[6] S. L. Palacios, A. Guinea, J.M. Fernández-Díaz, and R. D. Crespo, Phys. Rev. E60, R45 (1999). $\alpha_{6}=0$ does exist in addition to the kink-type solitarywave solution obtained by Agrawal and Headley [7].

\section{Acknowledgements}

This work was supported by the Korean Research Foundation of Korea, Grant No. 2002-015-CP0135. The author thanks Profs. Hasegawa and Agrawal for useful communications. The helpful comments by the anonymous referee are greatly appreciated.

[7] G. P. Agrawal and C. Headley III, Phys. Rev. A 46, 1573 (1992).

[8] G. P. Agrawal (private communication).

[9] M. Florjańczyk and L. Gagnon, Phys. Rev. A 41, 4478 (1990).

[10] F. M. Sears, L. G. Cohen, and J. Stone, J. Lightwave Technol. LT-2, 181 (1984).

[11] D. C. Calvo and T. R. Akylas, Phys. Rev. E56, 4757 (1997) and references therein.

[12] D. Anderson and M. Lisak, Phys. Rev. A 27, 1393 (1983). 